\title{
Rural-Urban Inequalities in Poor Self-Rated Health, Self-reported Functional Disabilities and Depression Among Chinese Older Adults: Evidence From the China Health and Retirement Longitudinal Study 2011 and 2015
}

\section{Haiting Jiang}

Nanjing Medical University https://orcid.org/0000-0003-2438-7230

\section{Bo Burström}

Karolinska Institutet

Jiaying Chen ( $\boldsymbol{\nabla}$ jychen@njmu.edu.cn )

Nanjing Medical University

Kristina Burström

Karolinska Institutet

\section{Research}

Keywords: China, depression, functional ability, health inequalities, household registration system, older adults, rural-urban, self-rated health, social determinants of health

Posted Date: November 10th, 2020

DOI: https://doi.org/10.21203/rs.3.rs-102597/v1

License: (c) (1) This work is licensed under a Creative Commons Attribution 4.0 International License. Read Full License

Version of Record: A version of this preprint was published at International Journal of Environmental Research and Public Health on June 18th, 2021. See the published version at https://doi.org/10.3390/ijerph18126557. 
1 Rural-urban inequalities in poor self-rated health,

2 self-reported functional disabilities and depression among

3 Chinese older adults: Evidence from the China Health and

4 Retirement Longitudinal Study 2011 and 2015

5 Authors:

6 Haiting Jiang ${ }^{1,2,3}$, Bo Burström ${ }^{2,4}$, Jiaying Chen ${ }^{1,2,5^{*}}$, Kristina Burström²,3,4

7 Affiliations:

8 1. School of Health Policy and Management, Nanjing Medical University,

9 No.101 Longmian Avenue, Nanjing 211166, P.R.China.

2. Centre for Health Policy Studies, Nanjing Medical University, No.101

11 Longmian Avenue, Nanjing 211166, P.R.China.

12 3. Health Outcomes and Economic Evaluation Research Group, Stockholm Centre for Healthcare Ethics, Department of Learning, Informatics, Management and Ethics, Karolinska Institutet, SE-17177 Stockholm, Sweden.

15 4. Equity and Health Policy Research Group, Department of Global Public Health, Karolinska Institutet, SE-17177 Stockholm, Sweden.

5. Institute of Healthy Jiangsu Development, No.101 Longmian Avenue, Nanjing 211166, P.R.China. 
Jiaying Chen, School of Health Policy and Management, Nanjing Medical University, No.101 Longmian Avenue, Nanjing 211166, P.R.China.

E-mail: jychen@njmu.edu.cn

\section{Abstract}

Background: Rural-urban inequalities in health among older adults in China have not been extensively studied from the national perspective. In particular, studies have not taken into account combinations of Hukou (household registration system in China) and actual residence, which may be important so as not to underestimate rural-urban differences. This study investigates rural-urban inequalities in prevalence of poor self-rated health, self-reported functional disabilities and depression among Chinese older adults in 2011 and 2015, and analyses sociodemographic determinants of rural-urban inequalities in self-reported health outcomes.

Methods: Data from the nationwide China Health and Retirement Longitudinal Study (CHARLS), on older adults aged 60 years and above in CHARLS 2011 and CHARLS 2015 were used. Only rural residents with rural Hukou and urban residents with urban Hukou were included. Sociodemographic factors, including age, sex, marital status, living arrangement, living near children, educational level and income were studied. Self-Rated Health (SRH) was assessed with a single question. Basic Activities of Daily Living (BADLs) and Instrumental Activities of Daily Living (IADLs) were used to measure self-reported functional abilities. The 10-item version of the Center for Epidemiologic Studies Depression Scale was used to measure self-reported 
depression.

Results: Rural respondents had poorer socioeconomic status and higher prevalence of poor SRH, functional disabilities and depression than urban respondents. The levels of functional disabilities, both BADLs and IADLs, were similar in 2011 and 2015, while the prevalence of poor SRH and self-reported depression was lower in 2015, both among rural and urban respondents. Impairments increased with age and appeared at younger age among rural respondents compared to urban respondents. Being female, unmarried, with low educational level and low income increased the odds ratios of reporting poor $\mathrm{SRH}$, functional disabilities and depression. Sensitivity analyses using only Hukou registration resulted in underestimation of rural-urban differences.

Conclusions: There were large rural-urban inequalities in poor SRH, self-reported functional disabilities and depression that were closely related to rural-urban differences in educational level and income.

Key words: China; depression; functional ability; health inequalities; household registration system; older adults; rural-urban; self-rated health; social determinants of health 


\section{Background}

The proportion of older adults in China is increasing [1,2]. By the end of 2018, there were 249 million people aged 60 and over years in China, accounting for $17.9 \%$ of the total population, and the population aged 65 and above reached 158 million. Life expectancy of Chinese people has been continuously increasing from 35.5 years in 1949 to 77.0 years in 2018 [3]. This may have a major impact on the coping capacities of China's health and social care systems.

Nearly $60 \%$ of the Chinese older adults live in rural areas [4]. Rural areas are less technically and economically developed and older adults remain at home while their children move to urban areas for work.[5]. Compared to older adults in urban areas, those in rural areas have lower socioeconomic status [6], less access to health services and social support [5] and report worse health status [7]. Disparities in living conditions between rural and urban areas might influence health outcomes of older adults. In addition, the household registration system (Hukou) in China, which classifies each person into a rural or an urban citizen, is a major means of monitoring population mobility and determining eligibility for state-provided services and welfare [8]. Citizens' interests and rights, such as education, health insurance, pension insurance, housing and employment, welfare and social security are determined by their Hukou registration [9]. As these rights differ between rural and urban areas, it may contribute to rural-urban inequalities in health outcomes $[10,11]$. In addition, a person with rural Hukou registration may live in an urban area and vice versa. Hence, when comparing rural and urban residents, using only the Hukou registration and not actual residence may cause misclassification. However, previous studies rarely take 
population mobility into consideration.

Different dimensions of health, such as functional abilities [18] and psychological health [19] are important for an individual's well-being [20]. Functional abilities deteriorate as people become older [21]. Furthermore, living alone or not [12-14] and the proximity to children could influence the older adults' health [13, 15]. Studies have shown that close proximity to children can be similar to co-residence [16], whereas others see any kind of non-co-residence as a sign of weakened inter-generational ties [17]. Socioeconomic factors may also be important determinants of health disparities among rural and urban older adults [7,22,23]. Few studies have compared the relationship between socioeconomic factors and self-reported health outcomes in Chinese older adults and examined whether rural-urban inequalities have changed over time.

The present study investigates rural-urban inequalities in 2011 and 2015 in the prevalence of poor self-rated health, self-reported functional disabilities and depression among Chinese older adults, and analyses sociodemographic determinants of rural-urban inequalities in those self-reported health outcomes.

\section{Methods}

\section{Data sources}

The China Health and Retirement Longitudinal Study (CHARLS) is a nationally representative household survey of the Chinese population [24]. It covers many aspects across the interviewee's lifetime, including household information, health 
status and functioning, health care and insurance, income, retirement and pension. The CHARLS survey was conducted by the National School of Development (China Centre for Economic Research) of Peking University. The survey used a face-to-face computer-assisted personal interview. All interviewees were required to sign informed consent. Ethical approval for the data collection in CHARLS was granted by the Ethical Review Committee of Peking University (IRB00001052-11015).

The baseline survey was conducted between June 2011 and March 2012 covering 28 provinces, 150 counties/districts, 450 communities, and 17,708 respondents (age $\geq 45$ years) from 10,257 households. Two follow-up interviews were conducted in 2013 and 2015. The sample was collected on a multi-stage probability-proportional-to-size technique, with county-level units stratified by region and then by urban districts (urban areas) or rural counties (rural area) [25] and per capita gross domestic product, with an overall response rate of $80.5 \%$ at the baseline [24]. Data are publicly available.

Our study used cross-sectional data of CHARLS 2011 and CHARLS 2015 to investigate the prevalence of poor self-rated health (SRH), impaired Basic Activities of Daily Living (BADLs) and impaired Instrumental Activities of Daily Living (IADLs) and self-reported depression among rural and urban Chinese older adults aged 60 years and above. There were 7,638 respondents in CHARLS 2011 and 10,185 respondents in CHARLS 2015, which were included in our study.

\section{Demographic indicators}


combined the information on Hukou registration with information on actual residence. Respondents, who have rural Hukou registration (agricultural household) and live in rural areas, were identified as rural respondents. Respondents, who have urban Hukou registration (non-agricultural household) and live in urban areas, were considered as urban respondents.

Age was the respondent's age in years at the time of the current wave's interview, calculated from the interview year and month minus the respondent's year and month of birth. Age was divided into age groups: $60-64,65-69,70-74,75-79,80-84,85-89$ and $90+$ years. Marital status was dichotomized into married and unmarried (i.e., widowed, never married, divorced or separated). Living arrangement was categorized into not living alone or living alone.

In CHARLS, respondents were asked whether they had a child who lives in the same city or county. Respondents co-residing with a child, or whose child lived in the same city or county were defined as "living near children". Respondents with children not co-residing nor living in the same city or county were defined as "not living near children". Respondents with no children were categorized as "no child".

\section{Socioeconomic indicators}

Educational level was based on highest self-reported attained education and categorized into below primary school, primary school, middle school, high school, and college and above. An individual's annual income was assessed by dividing the total household annual income by the number of persons living in the family within the last half-year, regardless of age and employment status [25]. Total household 
annual income is the sum of all income from all household members in the household including income from earnings, capital income, pension, government transfers and other income. Respondents were then ranked from lowest to highest by their annual income and divided into five groups of equal size. In CHARLS 2011, the lowest income group had an income below 610 Chinese Yuan (CNY); the second group from 611 to 2,100 CNY; the third group from 2,101 to 5,325 CNY; the fourth group from 5,326 to $12,067 \mathrm{CNY}$; the fifth and highest income group 12,068 CNY and above. In CHARLS 2015, the lowest income group had an income below $500 \mathrm{CNY}$; the second group from 501 to $1,169 \mathrm{CNY}$; the third group from 1,170 to 3,599 $\mathrm{CNY}$; the fourth group from 3,600 to 13,339 CNY; the fifth and highest income group 13,440 CNY and above.

\section{Outcome variables}

\section{Self-Rated Health (SRH)}

SRH is a generic measurement of health, which is widely used because of its simplicity and strong predictive power for future mortality [32,33]. Poor SRH has shown to predict increased long-term mortality in healthy, middle-aged individuals and in different socioeconomic groups [34]. SRH was measured by a single question. CHARLS adopted two different 5-point scales for self-reported general health, which were randomly assigned to participants to examine any effects of central tendency bias: i.e. that respondents either rate their health on the scale "excellent", "very good", "good", "fair" and "poor" or on the scale "very good", "good", "fair", "poor" and "very poor". Respondents were asked about their health status twice, once in the beginning of the health module with one scale and again at the end of the health 
module with the other scale. This study focused on persons with worse health outcomes, so the scale ranging from "very good" to "very poor" was used. Those who answered poor or very poor health were categorized as having poor health.

\section{Basic Activities of Daily Living (BADLs)}

Katz Activities of Daily Living Scale [26] was used to assess BADLs in CHARLS. It is a 6-item scale with dressing, bathing and showering, eating, getting in and out of bed, using the toilet, and controlling urination and defecation. Respondents were asked "Do you have any difficulty with the following basic activity of daily living?" With the scoring system used in CHARLS, each item was scored as following: 1 signified "do not have any difficulty", 2 signified "have difficulties but still can do it", 3 signified "have difficulties and help is needed", and 4 signified "cannot complete it". The Chinese version of the scale has been extensively tested and has been shown to yield reliable and valid responses [27]. The score of BADLs was calculated by the sum of all items. Having any difficulty with an activity (total score of BADLs $>6$ ) was identified as "impaired BADLs".

\section{Instrumental Activities of Daily Living (IADLs)}

IADLs are measured by the Lawton IADL Scale [28], which is ideal for community-dwelling older adults, as well as those who have been admitted to a hospital, short-term skilled nursing facility, or rehabilitation facility [29]. Performance was examined on the 5-items scale with doing household chores, cooking, shopping, managing money, taking medications [28], which are more complex levels of organized human behavior than BADLs [28]. Respondents were asked "Do you have 
any difficulty with the following instrumental activity of daily living?" With the scoring system used in CHARLS, each item was scored as following: 1 signified "do not have any difficulty", 2 signified "have difficulties but still can do it", 3 signified "have difficulties and help is needed", and 4 signified "cannot complete it". The sum score of all items $>5$ was categorized as "impaired IADLs".

\section{Self-reported depression}

In CHARLS, the 10-item version of the Center for Epidemiologic Studies Depression Scale (CES-D-10) was used to measure respondents' self-reported depression.

CES-D-10 score is the sum of the 10 self-reported questions, after reverse coding. Additionally, the scale for each of the ten questions was adjusted so that the response options were 0 to 3 , CES-D-10 ranges from 0 to 30 with higher scores indicating that the respondent felt more negatively during the past week. Each participant was categorized as having depressive symptoms if the total score of the CES-D-10 was 10 or above [30].

\section{Statistical analysis}

Respondents with missing data on Hukou registration, sex, marital status, living arrangement and educational level were excluded. In an initial step, analyses were performed on the prevalence rate of the health outcomes studied among respondents having different Hukou registration in rural and urban areas.

The main analyses in this study focus on rural respondents versus urban respondents. Hence, we excluded the respondents with rural Hukou registration living in urban 


\section{Results}

\section{Results of initial analyses on Hukou registration and residence}

areas and respondents with urban Hukou registration living in rural areas. Therefore, the final samples used for analyses were 6,048 respondents in CHARLS 2011 and 7,396 respondents in CHARLS 2015 (Figure 1).

Descriptive statistics were presented as means with standard deviation (SD) for numerical variables, or as percentages for categorical variables.

Differences in the prevalence of poor SRH, impaired BADLs, impaired IADLs and self-reported depression between the rural and urban respondents were examined separately for 2011 and 2015, by using Chi-square test or Fisher's Exact test for nominal categorical variables [31] and for variables with ordinal explanatory variables. Independent T-test was used to analyze the differences between rural and urban respondents in means of age and income. Multiple logistic regression models were performed to examine the over-risk of poor SRH, functional disabilities and depression among rural respondents and to what extent this over-risk was explained by sociodemographic determinants, adjusting for age and sex.

All statistical analyses were performed using SAS 9.4.1. The level of significance was specified at 0.05. A Bonferroni adjusted significance level was used in case of multiple tests.

\footnotetext{
Based on the initial analyses, the distribution of all respondents with different Hukou
} 
registration in rural and urban areas is shown in Supplementary Table S1. In 2011, $24 \%$ of those with rural Hukou registration lived in urban areas, and $11 \%$ of those with urban Hukou registration lived in rural areas. In 2015, these proportions were $26 \%$ and $14 \%$, respectively (Supplementary Table S1). Hence, in view of our objective to identify rural residents (with rural Hukou registration, living in rural areas) and urban residents (with urban Hukou registration, living in urban areas), some 35 to $40 \%$ of respondents were misclassified, if only Hukou registration had been used.

\section{Individual characteristics}

In the main analysis sample used in this study, the proportion of rural respondents was similar, nearly 73\%, in 2011 and 2015. Rural respondents were slightly younger than urban respondents. There were more females among rural respondents than urban respondents in 2015 (Table 1).

Compared to urban respondents, more rural respondents were unmarried. In 2011, nearly $10 \%$ of rural respondents and nearly $14 \%$ of urban respondents lived alone (compared to $9 \%$ rural respondents and $8 \%$ urban respondents in 2015). Fewer respondents lived near their children in 2015 than in 2011 (Table 1).

Rural respondents had lower levels of education and income than urban respondents.

In $201167 \%$ of the rural respondents had less than primary school, compared to $27 \%$ of urban respondents. In 2015, this proportion was slightly lower, among both rural and urban respondents. The mean individual annual income was about four times higher among urban respondents than among rural respondents, (Table 1). 


\section{Prevalence of health outcomes in 2011 and 2015}

Figure 2 shows the prevalence of all four health outcomes among rural and urban respondents, respectively, in 2011 and 2015. Rural respondents reported worse health outcomes than urban respondents. The prevalence of poor SRH and self-reported depression was statistically significantly lower in 2015 than in 2011, while the prevalence of impaired BADLs was significantly higher among both rural and urban respondents in 2015 than in 2011. There was no difference in the prevalence of impaired IADLs between the years among rural respondents, or among urban respondents.

Supplementary Table S2 also includes the results of the initial analyses of the prevalence rate of the health outcomes studied among respondents excluded from the main analyses, having discordant Hukou registration in rural and urban areas. The prevalence varied considerably between the different combinations of Hukou registration and actual residence. Respondents with rural Hukou registration who lived in urban areas had lower prevalence rate of the health outcomes than those living in rural areas. Respondents with urban Hukou registration living in rural areas had higher prevalence rate of the health outcomes than those living in urban areas, except impaired IADLs in 2011.

\section{Prevalence of poor SRH by individual characteristics}

Rural respondents reported higher prevalence of poor SRH than urban respondents (Table 2). The prevalence of poor SRH in 2015 was lower than in 2011, significantly declining from $39 \%$ to $30 \%$ among rural respondents and from $24 \%$ to $18 \%$ among 
urban respondents (Figure 2 and Table 2).

Rural respondents had significantly higher prevalence of poor SRH than urban respondents within each age group (Table 2). The youngest age group (60-64 years) of rural respondents had higher prevalence of poor SRH (36\%) than urban respondents aged $85-89$ years $(30 \%)$. The prevalence of poor SRH increased with age, except among respondents aged $90+$ years.

More females than males reported poor SRH, especially among rural respondents. Unmarried respondents had higher prevalence of poor SRH than married respondents. There was a gradient in poor SRH by education and income, with higher prevalence of poor SRH among respondents with lower educational level and lower income, both among rural and urban respondents (Table 2).

\section{Prevalence of impaired BADLs by individual characteristics}

The prevalence of impaired BADLs among the older respondents was slightly higher in 2015 than in 2011 (Supplementary Table S3). The prevalence of impaired BADLs increased with age. Compared to urban respondents, the impairments of BADLs appeared at younger age among rural respondents. In 2011, rural respondents aged 60-64 had higher prevalence of impaired BADL than urban respondents aged 75-79 years. There was no significant difference in the prevalence of impaired BADLs between rural and urban respondents who were aged 80 years and above. The prevalence of impaired BADLs was higher among females and respondents living alone than among males and respondents not living alone. In 2011, the prevalence of impaired BADLs among rural respondents who did not live near children was $32 \%$, 
compared to nearly $7 \%$ among urban respondents who did not live near children. However, in 2015, the prevalence of impaired BADLs among rural respondents was $26 \%$, while nearly $15 \%$ of urban respondents who did not live near children reported impaired BADLs.

The prevalence of impaired BADLs was lower among groups with higher educational level and higher income level. There was no significant difference in the prevalence of impaired BADLs between rural and urban respondents within each income group, except in the lowest income group in 2015 and the highest income group in both years (Supplementary Table S3).

\section{Prevalence of impaired IADLs by individual characteristics}

The prevalence of impaired IADLs among the older respondents was similar in 2011 and 2015 (Supplementary Table S4). The prevalence of impaired IADLs increased with age. Compared to urban respondents, the impairments of IADLs among rural respondents appeared at younger age. In 2015, rural respondents aged 65-69 years had higher prevalence (31\%) of impaired IADL than urban respondents aged 75-79 years (29\%). There was no significant difference in the prevalence of impaired BADLs between rural and urban respondents who were aged 85 years and above in 2011 or 2015. The prevalence of impaired IADLs was higher among females than males.

There was no significant difference between respondents living alone and not living alone. Compared to respondents who did not live near children, those living near children had higher prevalence of impaired IADLs. The respondents with no children reported higher prevalence of impaired IADLs. 
Groups with higher educational level and higher income level had a lower prevalence of impaired IADLs. There was no significant difference in the prevalence of impaired IADLs between rural and urban respondents within each income group, except in the lowest income group in 2015 and the highest income group in both years (Supplementary Table S4).

\section{Prevalence of self-reported depression by individual characteristics}

Compared to urban respondents, nearly twice as many rural respondents reported self-reported depression (Supplementary Table S5). The prevalence of self-reported depression among respondents was lower in 2015 than in 2011. The prevalence of self-reported depression was $47 \%$ in 2011 and $41 \%$ in 2015 among rural respondents, compared to $28 \%$ in 2011 and $23 \%$ in 2015 among urban respondents.

There was a significant difference of reporting depression between rural and urban respondents within each age group. Older respondents generally had higher prevalence of depression, but the prevalence was high also in the youngest age group of rural respondents. Respondents living alone, being unmarried, with lower educational level and lower income had a higher risk of reporting depression. More female than males reported depression, especially among rural respondents (Supplementary Table S5).

\section{Sociodemographic determinants of poor SRH, self-reported}

\section{functional disabilities and depression}

Multiple logistic regression analyses were done to estimate to what extent differences 
in sociodemographic determinants could explain rural-urban differences in health outcomes. After adjusting for age and sex, the difference in level of education and income between rural and urban respondents explained a large part of the differences in health outcomes between rural and urban respondents. Living alone or living near children was not significantly related to health outcomes.

As shown in Table 3a (2011) and 3b (2015), in Model 1, after adjusting for age and sex, rural respondents had a higher odds ratio of reporting poor SRH in 2011 $(\mathrm{OR}=2.05)$ and in $2015(\mathrm{OR}=2.00)$. Further adjustment for marital status, living arrangement, living near children (Model 2) did not significantly change the odds ratio for rural respondents. In Model 3, further adjustment for education level reduced the odds ratio for having poor SRH among rural respondents in $2011(\mathrm{OR}=1.66)$ and in $2015(\mathrm{OR}=1.74) .$. In 2011, when instead adjusting for income group (Model 4) the odds ratio was lower $(\mathrm{OR}=1.55)$ (Table 3a). In the full model for 2011 (Model 5), the odds ratio for having poor SRH was further reduced (OR=1.34). In 2015, the proportion of respondents with missing information of income was more than $50 \%$; hence income was not included in the regression analysis.

Similar findings were seen in regression analyses for impaired BADL, impaired IADL and self-reported depression (Supplementary Tables S6, S7 and S8, respectively). For impaired BADLs, in Model 1, rural respondents had higher odds ratio of having impaired BADLs in 2011(OR=1.99) than in $2015(\mathrm{OR}=1.76)$. In 2011, adjusting for living arrangement, live near children, educational level and income group, in Model 5, reduced the odds ratio for having impaired BADLs to 1.09 (Table S6).

For impaired IADLs, in Model 1, the odds ratio of rural respondents reporting 
impaired IADLs was 2.05 in 2011 and 2.03 in 2015. In 2011, adjusting for living arrangement, live near children, educational level and income group, in Model 5, reduced the odds ratio for reporting impaired IADLs to 1.13 (Table S7).

For self-reported depression, in Model 1, rural respondents had higher odds ratio $(\mathrm{OR}=2.36)$ of reporting self-reported depression in 2011 and in $2015(\mathrm{OR}=2.32)$. In 2011, adjusting for living arrangement, live near children, educational level and income group, in Model 5, reduced the odds ratio for having self-reported depression to 1.38 (Table S8).

\section{Discussion}

This study found substantially higher rates of adverse health outcomes among rural than urban respondents, both in 2011 and 2015. The prevalence rate of poor SRH, impaired IADLs and self-reported depression was lower in 2015 than in 2011, but the prevalence rate of impaired BADLs was higher in 2015 than in 2011. However, the rural-urban differences were similar in both years. A novel classification, combining Hukou registration with actual residence, was used to distinguish rural respondents from urban respondents. The usual classification, using only Hukou registration and not actual residence, resulted in smaller rural-urban differences.

Rural respondents with lower socioeconomic status had statistically significantly higher prevalence of poor SRH, self-reported impaired BADL and IADL, and self-reported depression than urban respondents, which is in line with other studies [7, 
$22,25]$. It is also consistent with lower life expectancy in rural compared to urban areas [35]. In 2010, the life expectancy in Beijing and Shanghai was 80.2 years, compared to 72.2 years in Gansu province [36].

Our results show that rural respondents have worse SRH than urban respondents, which is consistent with worse functional abilities, psychological health conditions and socioeconomic status.

Functional ability, the fundamental capability of individuals, is related to successful and positive ageing [37]. Overall, difficulties in performing daily activities, both in BADLs and IADLs, were strongly associated with age, and these impairments appeared at substantially younger age among rural respondents than urban respondents. This may indicate that the need of assistance because of functional limitations begins at an earlier age among rural residents.

There are different assessments for functional ability. Our study showed that the prevalence of impaired IADLs was higher than the prevalence of impaired BADLs among the rural respondents in both years, and slightly higher in 2015 than in 2011. Most studies assess functional abilities with BADLs [38-40], measured with basic activities, such as eating and toileting. BADLs have an inherent hierarchical structure intended to reflect primary biological and psychosocial function [41] or, as alternately described, primary sociobiological function, which is related to personal care, most 
often used for measuring the functional ability. The measure IADLs is considered to handle that complex functions normally are lost before BADLs [29], and necessary for functioning in community settings. Therefore, assessing IADLs may identify incipient decline in older adults or other respondents who are otherwise capable and healthy [29]. However, few studies include IADLs in the concept of functional disability with BADLs [42-44]. Taking both into consideration may provide an overall assessment of the functional abilities among the older adults. Respondents with worse health are now likely to survive longer than they would have in the past, resulting in populations of older respondents with higher rates of disabilities at older age.

In our study, rural older adults had nearly two times higher prevalence of self-reported depression than urban older adults, which is similar to other studies [45], The prevalence was lower in 2015 than in 2011. The improvement in psychological health might be related to increasing income. It might also be related to improved health care access, social support and participation in the society [46], however this was not investigated in the present study.

We hypothesized that marital status would show a positive association with SRH, functional abilities and self-reported depression. This was also seen in our study, as in other studies [47, 48]. There might be several explanations for this, such as a direct health promotional effect of the marriage and social support from spouse [49]. In 
addition, many Chinese older adults live with their children and not alone. Previous studies have concluded that living alone is an independent risk factor contributing to adverse health outcomes among Chinese older adults, especially among females [50, 51], and that younger adults were less likely to work as migrants when their parents were ill or in poor health status [52]. However, in the present study, rural-urban inequalities in self-reported health outcomes were not associated to living arrangements, nor to whether living near children.

The main focus of our study was on rural-urban inequalities in health outcomes.

These health inequalities were considerable, with two-fold odds ratios of poor SRH, impaired BADL, impaired IADL and depression among rural compared to urban residents. The health inequalities reflect and are closely related to large differences in the level of education and income between rural and urban areas. Urban respondents had four times higher income than rural residents, and two-thirds of rural respondents had below primary school, compared to one-fourth in urban areas. The rural-urban inequalities in poor SRH, functional disabilities and self-reported depression were to a large extent explained by rural-urban inequalities in educational level and income. In 2011, especially the rural/ urban differences in income explained a large part of the rural-urban differences in health outcomes. Due to the large number with missing data on income in 2015, the role of income could not be studied.

\section{Strengths and limitations}


A major strength of our study is that it is the first study that limited the samples to respondents with rural Hukou living in rural areas and respondents with urban Hukou living in urban areas in order to control the influence of population mobility.

Respondents with different Hukou are covered by different insurance and health care. In CHARLS, we found that among respondents with rural Hukou, almost 25\% lived in urban areas, and more than $10 \%$ of respondents with urban Hukou lived in rural areas. In addition, there was a significant difference in the health outcomes between the respondents who lived in the same area but with different Hukou. This underlines the importance of taking not only Hukou registration but also actual residence into account, when comparing rural and urban residents. Using only Hukou registration underestimated the differences between rural and urban respondents.

Another strength is that we used a nationwide representative sample derived from the large and diverse population in China to study the rural-urban inequalities in self-reported health outcomes and investigate different dimensions of self-reported health status, including general health, functional abilities and psychological health. However, this study also has several limitations. Some factors were not included in our study. We were not able to explore the effects of other sociocultural and environmental factors due to lack of specific data. Unfortunately, the importance of level of income could not be studied in 2015 because of the large proportion (nearly $60 \%$ ) with missing data on income in 2015. In addition, this study shows only a four 
year difference, which might be not long enough time to show a trend, but it might also contribute to the sparse literature on whether rural-urban inequalities have changed over the years. The cross-sectional design of the study prevents causal inference.

Nevertheless, our study indicates that there are important differences in self-reported health, functional disabilities and self-reported depression between rural and urban older adults, which to a great extent were explained by rural-urban inequalities in educational level and income, which are central determinants of health. Redistribution of income from rich to poor may be one of the most important policy levers for improving population health [36]. Welfare state policies and interventions often aim at addressing important determinants of health [53]. Our results may provide a basis for interventions to improve health and functional abilities among older people, addressing differences in demographics and socioeconomic conditions between rural and urban areas. Further studies should investigate how health and social services might be strengthened in rural areas, in order to improve health and functional abilities among rural older adults.

\section{Conclusions}

Using a novel classification, combining Hukou registration and actual residence, to distinguish rural and urban residents, we found large rural-urban inequalities in poor 

which were to a great extent associated to rural-urban inequalities in educational level and income. Rural respondents had higher prevalence of adverse health outcomes and the impairment appeared at younger age among rural than urban respondents. Our results may provide a basis for interventions to improve health and functional abilities among older people, addressing differences in demographic and socioeconomic conditions and access to health and social services between rural and urban areas.

\section{Abbreviations}

492 CHARLS: China Health and Retirement Longitudinal Study

493 SRH: Self-Rated Health

494

BADLs: Basic Activities of Daily Living

495

IADLs: Instrumental Activities of Daily Living

496

CNY: Chinese Yuan

497

OR: Odds Ratio

SD: Standard Deviation 
500 Ethics approval and consent to participate

501 All interviewees were required to sign informed consent. Ethical approval for the data

502 collection in CHARLS was granted by the Ethical Review Committee of Peking

503 University (IRB00001052-11015).

\section{Consent for publication}

505 Not applicable.

506 Availability of data and materials

507 The CHARLS dataset is publicly available. Information about the data source and

508 available data are found at http://charls.pku.edu.cn/pages/data/111/en.html.

509 Researchers can obtain these data after submitting a data use agreement to the

510 CHARLS team.

\section{Competing interests}

512 The authors declare that they have no competing interests.

\section{$513 \quad$ Funding}

514 This study was funded by the National Natural Science Foundation of China under 


\section{Authors' contributions}

HJ and KB conceived the study. All authors contributed to the design. HJ carried out the preliminary analyses under the supervision of KB. All authors contributed to the interpretation of results. $\mathrm{HJ}$ drafted the first version of the work. $\mathrm{BB}, \mathrm{JC}$ and $\mathrm{KB}$ revised the manuscript critically for important intellectual content. All authors read and approved the final manuscript submitted for publication.

\section{Acknowledgments}

Data used in this study were provided by the China Health and Retirement Longitudinal Study (CHARLS).

\section{References}

1. Stewart Williams J, Norström F, Ng N. Disability and ageing in China and India - decomposing the effects of gender and residence. Results from the WHO study on global AGEing and adult health (SAGE). BMC Geriatrics. 2017; 17(1): 197 .

2. Liang Y, Welmer AK, Wang R, Song A, Fratiglioni L, Qiu C. Trends in incidence of disability in Activities of Daily Living in Chinese older adults: 1993-2006. Journal of the American Geriatrics Society. 2017; 65(2):306-312.

3. National Health Commission of the People's Republic of China. China Public Health Statistical Yearbook (2018): Peking Union Medical College Press. 2019. 
4. Wang Q, Zhang X, Fang L, Guan Q, Guan L, Li Q. Prevalence, awareness, treatment and control of diabetes mellitus among middle-aged and elderly people in a rural Chinese population: A cross-sectional study. PloS One. 2018; 13(6):e0198343.

5. Zimmer Z, Kwong J. Socioeconomic status and health among older adults in rural and urban China. Journal of Aging \& Health. 2004; 16(1):44-70.

6. Zhang Y, Goza FW. Who will care for the elderly in China? A review of the problems caused by China's one-child policy and their potential solutions. Journal of Aging Studies. 2006; 20(2):151-164.

7. Sun S, Chen J, Johannesson M, Kind P, Xu L, Zhang Y, Burström K. Regional differences in health status in China: Population health-related quality of life results from the National Health Services Survey 2008. Health \& Place. 2011; 17(2):671-680.

8. Chan KW. The household registration system and migrant labor in China: notes on a debate. Population and Development Review. 2010; 36(2):357-364.

9. Lu Y. Does hukou still matter? The household registration system and its impact on social stratification and mobility in China. Social Sciences in China. 2008; 29(2):56-75.

10. Liu H, Rizzo JA, Fang H. Urban-rural disparities in child nutrition-related health outcomes in China: The role of hukou policy. BMC Public Health. 2015; 15(1):1-11.

11. Chan $\mathrm{KW}$, Zhang L. The Hukou system and rural-urban migration in China: Processes and changes. Chinese Sociology \& Anthropology. 1996; 29(1):15-26.

12. Magaziner J, Cadigan DA, Hebel JR, Parry RE. Health and living arrangements among older women: Does living alone increase the risk of illness. Journal of Gerontology. 1988; 43(5):M127-M133.

13. Sereny M. Living arrangements of older adults in China: The interplay among preferences, realities, and health. Research on Aging. 2011; 33(2):172-204.

14. Hawton A, Green C, Dickens AP, Richards SH, Taylor RS, Edwards R, Greaves CJ, Campbell JL. The impact of social isolation on the health status and health-related quality of life of older people. Quality of Life 
Research. 2011; 20(1):57-67.

15. Silverstein $\mathrm{M}$, Cong $\mathrm{Z}$, Li S. Intergenerational transfers and living arrangements of older people in rural China: Consequences for psychological well-being. The Journals of Gerontology Series B: Psychological Sciences and Social Sciences. 2006; 61(5):S256-S266.

16. Bian F, Logan JR, Bian Y. Intergenerational relations in urban China:

17. Hank K. Proximity and contacts between older parents and their children: A European comparison. Journal of Marriage and Family. 2007; 69(1): 157-173.

18. Bernard SL, Kincade JE, Konrad TR, Arcury TA, Rabiner DJ, Woomert A, DeFriese GH, Ory MG. Predicting mortality from community surveys of older adults: the importance of self-rated functional ability. The Journals of Gerontology Series B: Psychological Sciences and Social Sciences. 1997; 52(3):S155-S163.

19. Bowling A, Iliffe S. Psychological approach to successful ageing predicts future quality of life in older adults. Health and Quality of Life Outcomes. 2011; 9(1): 13 .

20. Phelan EA, Anderson LA, Lacroix AZ, Larson EB. Older adults' views of "successful aging"-how do they compare with researchers' definitions? Journal of the American Geriatrics Society. 2004; 52(2):211-216.

21. McPhee JS, French DP, Jackson D, Nazroo J, Pendleton N, Degens H. Physical activity in older age: perspectives for healthy ageing and frailty. Biogerontology. 2016; 17(3):567-580.

22. Woolf SH, Braveman P. Where health disparities begin: the role of social and economic determinants - and why current policies may make matters worse. Health Affairs. 2011; 30(10):1852-1859.

23. Smith KV, Goldman N. Socioeconomic differences in health among older adults in Mexico. Social Science \& Medicine. 2007; 65(7):1372-1385.

24. Zhao Y, Hu Y, Smith JP, Strauss J, Yang G. Cohort profile: the China Health and Retirement Longitudinal Study (CHARLS). International Journal of Epidemiology. 2014; 43(1):61.

25. Sun S, Chen J, Johannesson M, Kind P, Xu L, Zhang Y, Burström K. 
Population health status in China: EQ-5D results, by age, sex and socio-economic status, from the National Health Services Survey 2008. Quality of Life Research. 2011; 20(3):309-320.

26. KATZ, SIDNEY. Assessing Self-maintenance: Activities of Daily Living, Mobility, and Instrumental Activities of Daily Living. Journal of the American Geriatrics Society. 1983; 31(12):721-727.

27. Gu D, Zeng Y. Sociodemographic Effects on the Onset and Recovery of ADL Disability among Chinese Oldest-old. Demographic Research. 2004; 11(1):1-42.

28. Lawton MP, Brody EM. Assessment of older people: self-maintaining and instrumental activities of daily living. The Gerontologist. 1969; 9(3):179-186.

29. Graf $\mathrm{C}$. The Lawton instrumental activities of daily living scale. The American Journal of Nursing. 2008; 108(4):52-62; 62-53.

30. Andresen EM, Malmgren JA, Carter WB, Patrick DL. Screening for depression in well older adults: evaluation of a short form of the CES-D (Center for Epidemiologic Studies Depression Scale). American Journal of Preventive Medicine. 1994; 10(2):77-84.

31. Chapman D, Nam J-m. Asymptotic power of chi square tests for linear trends in proportions. Biometrics. 1968; 24(2):315-327.

32. Dowd JB, Zajacova A. Does self-rated health mean the same thing across socioeconomic groups? Evidence from biomarker data. Annals of Epidemiology. 2010; 20(10):743-749.

33. Jylhä M. What is self-rated health and why does it predict mortality? Towards a unified conceptual model. Social Science \& Medicine. 2009; 69(3):307-316.

34. Burstrom B, Fredlund P. Self rated health: Is it as good a predictor of subsequent mortality among adults in lower as well as in higher social classes? Journal of Epidemiology and Community Health. 2001; 55(11):836-840.

35. Gui S, Chen J . Average life expectancy of the Chinese population in 1949-2019: trends, contributors and prospects[J]. China Population and Development Studies, 2020, 3(2):142-153. 
36. Luo W, Xie Y . Economic Growth, Income Inequality And Life Expectancy In China[J]. Social Science \& Medicine, 2020, 256:113046.

37. Bowling A. The concepts of successful and positive ageing. Family Practice. 1993; 10(4):449-453.

38. Iwarsson S, Isacsson $\AA$, Lanke J. ADL dependence in the elderly population living in the community: the influence of functional limitations and physical environmental demand. Occupational Therapy International. 1998; 5(3):173-193.

39. Johnson JK, Lui L-Y, Yaffe K. Executive function, more than global cognition, predicts functional decline and mortality in elderly women. The Journals of Gerontology Series A: Biological Sciences and Medical Sciences. 2007; 62(10):1134-1141.

40. Gubhaju B, Østbye T, Chan A. Living arrangements of community-dwelling older Singaporeans: predictors and consequences. Ageing \& Society 2017; 38(6):1174-1198.

41. Katz S, Ford AB, Moskowitz RW, Jackson BA, Jaffe MW. Studies of Illness in the Aged: The Index of ADL: A Standardized Measure of Biological and Psychosocial Function. JAMA 1963; 185(12):914-919.

42. Ouchi Y, Kato Y, Nakai M, Ishikawa H, Nakamura K, Nakatsuka M, Kasai M, Tanaka N, Meguro K. Scope (quantitative) and performance (qualitative) of instrumental ADLs in older adults with mild cognitive impairment: Difference between very mild Alzheimer's disease and very mild subcortical vascular dementia - The Kurihara Project - Alzheimer's \& Deme. Alzheimers \& Dementia the Journal of the Alzheimers Association. 2012; 8(4):P318-P318.

43. Martin LG, Feng Q, Schoeni RF, Yi Z. Trends in functional and activity limitations among Chinese oldest-old, 1998 to 2008. Population \& Development Review. 2014; 40(3):475-495.

44. Fitz AG, Teri L. Depression, cognition, and functional ability in patients with Alzheimer's disease. Journal of the American Geriatrics Society. 1994; 42(2):186-191.

45. Purtle J, Nelson KL, Yang Y, Langellier B, Stankov I, Roux AVD. Urban-rural differences in older adult depression: A systematic review and meta-analysis of comparative studies. American Journal of Preventive 
Medicine. 2019; 56(4):603-613.

672

673

46. Fulbright SA. Rates of depression and participation in senior centre activities in community-dwelling older persons. Journal of Psychiatric and Mental Health Nursing. 2010; 17(5):385-391.

47. Stutzer A, Frey BS. Does marriage make people happy, or do happy people get married? The Journal of Socio-Economics. 2006; 35(2):326-347.

48. Perkins JM, Lee H-y, James K, Oh J, Krishna A, Heo J, Lee J-k, Subramanian S: Marital status, widowhood duration, gender and health outcomes: a cross-sectional study among older adults in India. BMC Public Health. 2016; 16(1): 1032 .

49. Goodwin JS, Hunt WC, Key CR, Samet JM. The effect of marital status on stage, treatment, and survival of cancer patients. JAMA. 1987; 258(21):3125-3130.

50. Mui AC. Living alone and depression among older Chinese immigrants. Journal of Gerontological Social Work. 1999; 30(3-4):147-166.

51. Chou K-L, Ho A, Chi I. Living alone and depression in Chinese older adults. Aging and Mental Health. 2006; 10(6):583-591.

52. Giles J, Mu R. Elderly parent health and the migration decisions of adult children: Evidence from rural China. Demography. 2007; 44(2):265-288.

53. Marmot M, Allen J, Bell R, Bloomer E, Goldblatt P. WHO European review of social determinants of health and the health divide. Lancet. 2012; 380(9846):1011-1029. 


\section{CHARLS 2011 CHARLS 2015}

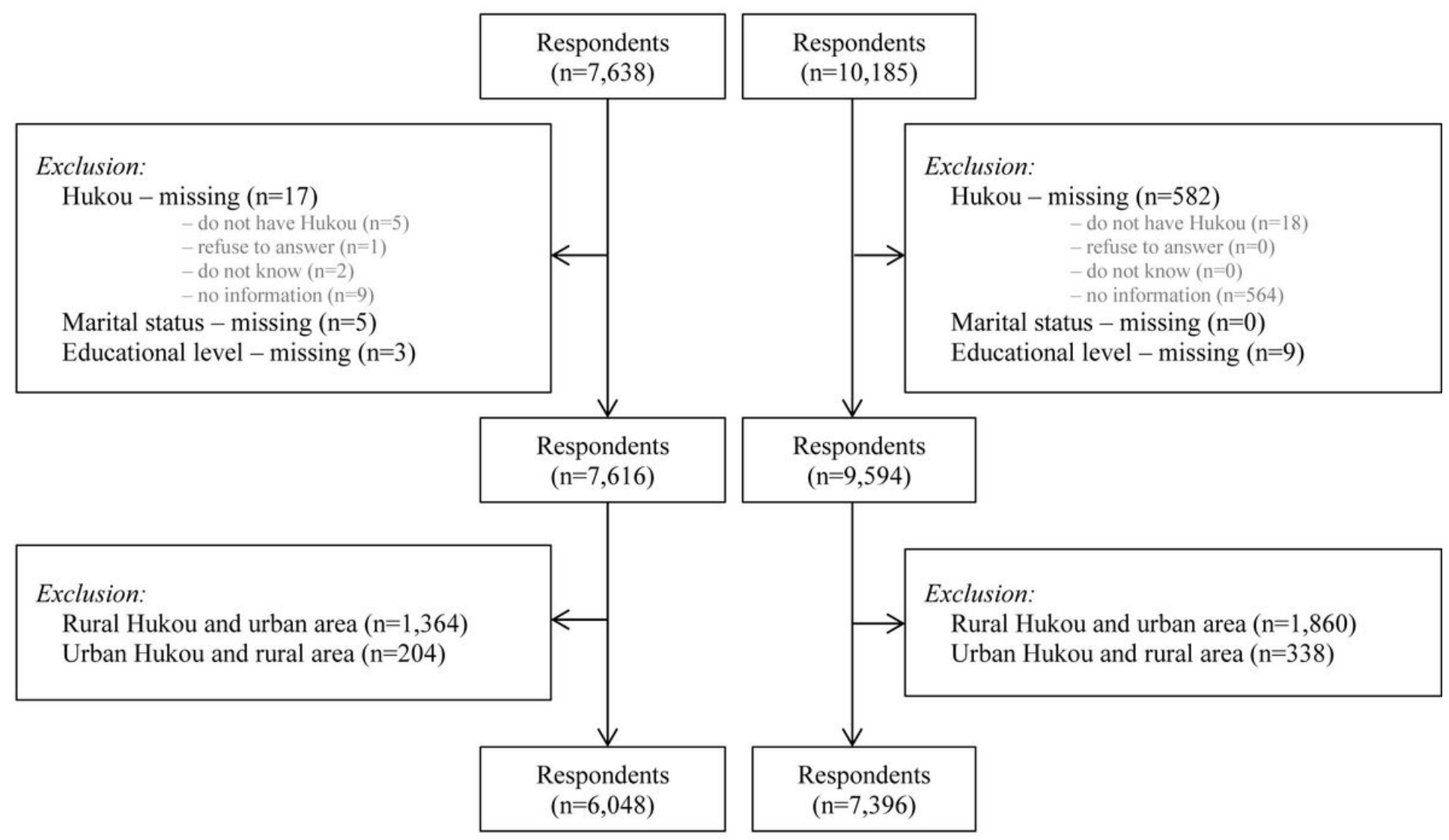

\section{Figure 1}

Flow diagram of samples, CHARLS 2011 and CHARLS 2015 


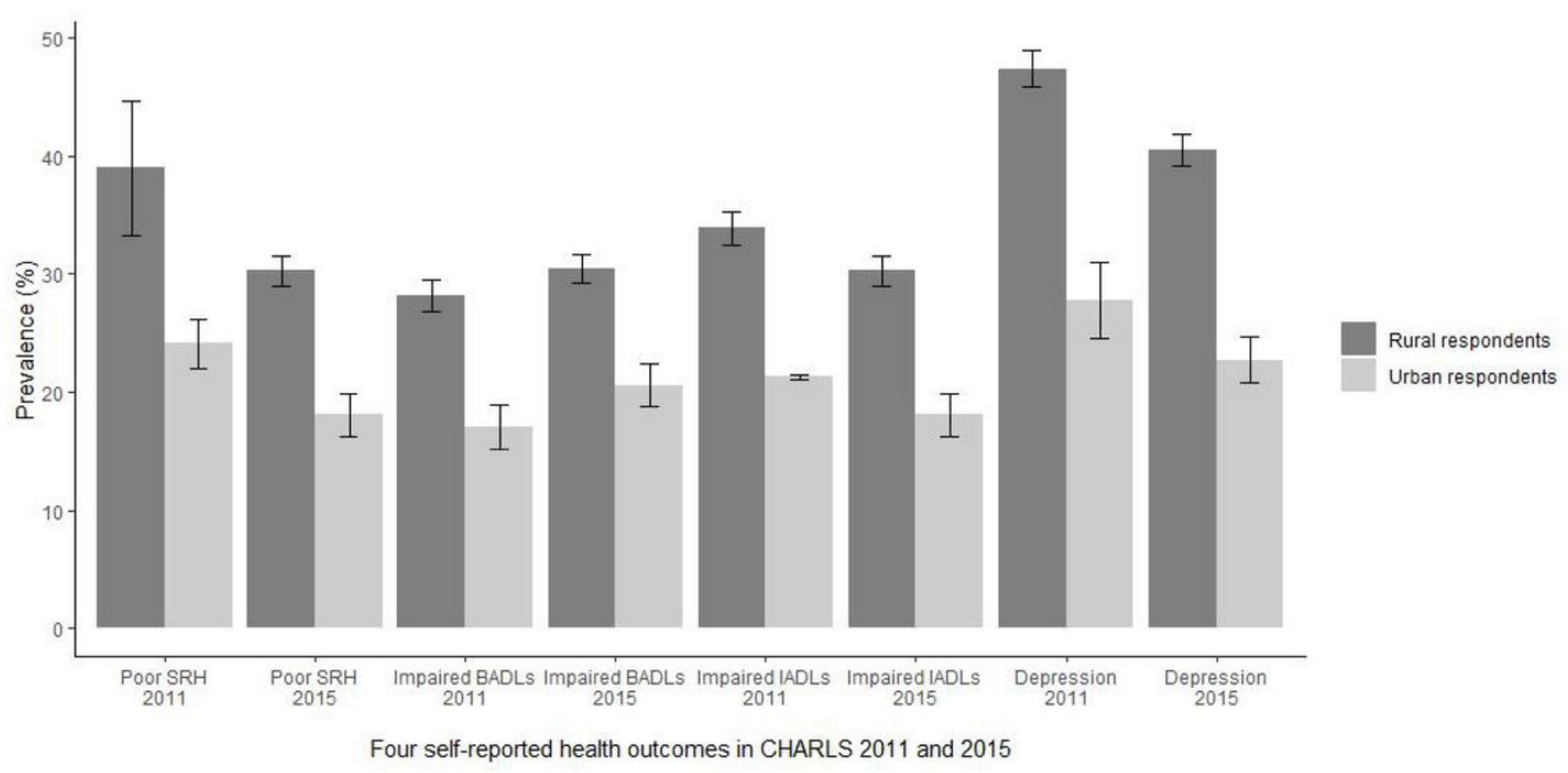

Figure 2

Prevalence (\%) of poor Self-Rated Health (SRH), impaired Basic Activities of Daily Living (BADLs), impaired Instrumental Activities of Daily Living (IADLs) and self-reported depression among rural and urban respondents, CHARLS 2011 and CHARLS 2015

\section{Supplementary Files}

This is a list of supplementary files associated with this preprint. Click to download.

- RuralurbaninequalitiesCHARLSTables201031.pdf

- RuralurbaninequalitiesCHARLSSupplementary201031.pdf 